\title{
Practical guidelines for managing adults with 22q11.2 deletion syndrome
}

\author{
Wai Lun Alan Fung, MD, ScD ${ }^{1-4}$, Nancy J. Butcher, MSc, ${ }^{2,5}$, Gregory Costain, PhD ${ }^{2,5}$, \\ Danielle M. Andrade, MD, MSc ${ }^{1,6}$, Erik Boot, MD, PhD ${ }^{1-4,7}$, Eva W.C. Chow, MD, FRCPC ${ }^{2,4}$, \\ Brian Chung, MRCPCH, MBBS ${ }^{8}$, Cheryl Cytrynbaum, MS, $\mathrm{CGC}^{9}$, Hanna Faghfoury, MD ${ }^{10}$, \\ Leona Fishman, MD, FRCPC ${ }^{9}$, Sixto García-Miñaúr, MD ${ }^{11}$, Susan George, MD, FRCPC1,12,13, \\ Anthony E. Lang, MD, FRCPC ${ }^{6,14}$, Gabriela Repetto, MD ${ }^{15}$, Andrea Shugar, MS, CGC ${ }^{9}$, \\ Candice Silversides, MD, FRCPC ${ }^{1,16,17}$, Ann Swillen, PhD ${ }^{18,19}$, Therese van Amelsvoort, MD, PhD ${ }^{20}$, \\ Donna M. McDonald-McGinn, MS, CGC ${ }^{21-23}$ and Anne S. Bassett, MD, FRCPC $1-5,12,17$
}

22q11.2 Deletion syndrome (22q11.2DS) is the most common microdeletion syndrome in humans, estimated to affect up to 1 in 2,000 live births. Major features of this multisystem condition include congenital anomalies, developmental delay, and an array of early- and later-onset medical and psychiatric disorders. Advances in pediatric care ensure a growing population of adults with 22q11.2DS. Informed by an international panel of multidisciplinary experts and a comprehensive review of the existing literature concerning adults, we present the first set of guidelines focused on managing the neuropsychiatric, endocrine, cardiovascular, reproductive, psychosocial, genetic counseling, and other issues that are the focus of attention in adults with 22q11.2DS. We propose practical strategies for the recognition, evaluation, surveillance, and management of the associated morbidities.

Genet Med advance online publication 8 January 2015

Key Words: 22q11.2 deletion; clinical practice guidelines; DiGeorge syndrome; treatment; velocardiofacial syndrome
For individuals with 22q11.2 deletion syndrome (22q11.2DS) (OMIM 188400/192430), the most common microdeletion syndrome in humans, ${ }^{1-4}$ survival to adulthood is now the norm. ${ }^{2}$ In addition to the well-known congenital and developmental features, ${ }^{1,2}$ treatable later-onset conditions are increasingly recognized as important components of 22q11.2DS. The multisystem nature and associated burden of morbidities means that adults with 22q11.2DS may be seen in virtually any medical practice. ${ }^{1}$ Chromosome $22 \mathrm{q} 11.2$ deletions are absent in large populations of healthy controls, implying a high collective penetrance for at least one major phenotypic feature. ${ }^{5}$ The growing numbers of affected children advancing to adulthood and the advent of noninvasive prenatal testing for 22q11.2 deletions have prompted demands for information about longer-term issues. The existing clinical practice guidelines for 22q11.2DS are focused primarily on children. ${ }^{1}$ We therefore present the first set of guidelines focused on managing the neuropsychiatric, endocrine, cardiovascular, reproductive, psychosocial, genetic counseling, and other issues pertinent to adults with 22q11.2DS. A comprehensive review of the existing literature concerning adults was complemented by the collective experience of professionals from various disciplines dedicated to caring for a total of more than 500 adults with 22q11.2DS.

\footnotetext{
The first three authors contributed equally to this work.

${ }^{1}$ The Dalglish Family Hearts and Minds Clinic for 22q11.2 Deletion Syndrome, Toronto General Hospital, University Health Network, Toronto, Ontario, Canada; ${ }^{2}$ Clinical Genetics Research Program, Centre for Addiction and Mental Health, Toronto, Ontario, Canada; ${ }^{3}$ Department of Psychiatry and Toronto General Research Institute, University Health Network, Toronto, Ontario, Canada; ${ }^{4}$ Department of Psychiatry, University of Toronto, Toronto, Ontario, Canada; ${ }^{5}$ Institute of Medical Science, University of Toronto, Toronto, Ontario, Canada; ${ }^{6}$ Division of Neurology, Toronto Western Hospital, Krembil Neurosciences Centre, University of Toronto, Toronto, Ontario, Canada; ${ }^{7}$ Department of Nuclear Medicine, Academic Medical Center, Amsterdam, The Netherlands; ${ }^{8}$ Department of Paediatrics and Adolescent Medicine, LKS Faculty of Medicine, The University of Hong Kong, Hong Kong Special Administrative Region, China; ${ }^{9}$ Division of Clinical and Metabolic Genetics, The Hospital for Sick Children, Toronto, Ontario, Canada; ${ }^{10}$ The Fred A. Litwin and Family Centre in Genetic Medicine, University Health Network and Mount Sinai Hospital, Toronto, Ontario, Canada; ${ }^{11}$ Institute of Medical and Molecular Genetics, Hospital Universitario La Paz, Universidad Autónoma de Madrid, IdiPAZ, Madrid, Spain; ${ }^{12}$ Campbell Family Mental Health Research Institute, Centre for Addiction and Mental Health, Toronto, Ontario, Canada; ${ }^{13}$ Department of Pharmacology, University of Toronto, Toronto, Ontario, Canada; ${ }^{14}$ The Morton and Gloria Shulman Movement Disorders Clinic and the Edmond J. Safra Program in Parkinson's Disease, Toronto Western Hospital, Toronto, Ontario, Canada; ${ }^{15}$ Center for Genetics and Genomics, Facultad de Medicina, Clínica Alemana Universidad del Desarrollo, Santiago, Chile; ${ }^{16}$ Division of Obstetric Medicine, Medical Disorders of Pregnancy Program, Mount Sinai Hospital, Toronto, Ontario, Canada; ${ }^{17}$ Division of Cardiology, Department of Medicine, University Health Network, Toronto, Ontario, Canada; ${ }^{18}$ Center for Human Genetics, University Hospital Gasthuisberg, Leuven, Flanders, Belgium; ${ }^{19}$ Department of Human Genetics, University of Leuven (KU Leuven), Leuven, Flanders, Belgium; ${ }^{20}$ Department of Psychiatry and Psychology, School for Mental Health and Neuroscience, Maastricht University Medical Centre, Maastricht, The Netherlands; ${ }^{21}$ Division of Human Genetics, $22 \mathrm{q}$ and You Center, The Children's Hospital of Philadelphia, Philadelphia, Pennsylvania, USA; ${ }^{22}$ Clinical Genetics Center, The Children's Hospital of Philadelphia, Philadelphia, Pennsylvania, USA; ${ }^{23}$ Department of Pediatrics, Perelman School of Medicine, University of Pennsylvania, Philadelphia, Pennsylvania, USA. Correspondence: Anne S. Bassett (anne.bassett@utoronto.ca)
} 


\section{METHODS}

These guidelines were developed in two stages. First, we conducted a comprehensive review of the existing literature. The EMBASE (1947 to week 28 of 2013), MEDLINE (1946 to 16 July 2013), and PsycINFO (1806 to the second week of July 2013) databases were searched on 16 July 2013 using the OVID interface and the search term "[ $\left(22 \mathrm{q}^{*}\right.$ adj3 deletion $)$ OR (velocardiofacial OR velocardio-facial OR velo-cardiofacial OR velo-cardio-facial OR VCFS) OR (DiGeorge syndrome) OR (conotruncal anomaly face syndrome)] AND adult." This search was limited to the literature concerning humans; it yielded 1,785 potentially relevant articles (including journal articles, conference proceedings, book chapters, and books). Initial screening by scanning titles resulted in the elimination of 718 duplicates and 392 irrelevant articles, leaving 675 articles. Screening abstracts resulted in the exclusion of a further 176 articles. A manual search of the remaining 499 articles resulted in the elimination of 261 without a substantive focus on adult issues in 22q11.2DS. Ten articles of potential interest were unavailable (nine case reports and one case series involving three patients). The search was repeated on 8 July 2014 and resulted in the identification of 25 additional articles, for a final total of 253.

Second, a draft consensus document was created by clinicians and researchers based in Toronto (Canada) who have diverse expertise in managing adult issues in 22q11.2DS. The resulting manuscript was then circulated among seven clinicians/researchers from Asia, Europe, South America, and the United States who are experienced in the clinical care of and/or research on adults with 22q11.2DS for additional feedback. All authors met at least one of the following criteria: (i) substantial clinical experience working with adults with 22q11.2DS (operationalized as having seen at least 10 adult patients with 22q11.2DS in both consultation and follow-up) and (ii) substantial research experience with adults with 22q11.2DS (based on peer-reviewed publications). The results of the literature search were used to guide the discussion and to support consensus recommendations with scientific evidence when possible. A relatively limited literature about this complex condition in adulthood exists, however, particularly for management issues. Consequently, as for the general clinical practice guidelines for 22q11.2DS, ${ }^{1}$ virtually all of the evidence is level III or IV (e.g., descriptive studies, case series, expert opinions). Thus we did not formally grade the individual recommendations presented.

\section{REVIEW AND PRACTICE GUIDELINES}

The phenotypes associated with 22q11.2DS are highly variable in number and severity, even within families and between monozygotic twins. ${ }^{6}$ This variability contributed to the historical naming and characterization of what were originally thought to be distinct clinical syndromes (e.g., DiGeorge syndrome, velocardiofacial syndrome) before the discovery of a common underlying microdeletion on chromosome 22q11.2. The available information on the adult phenotype and natural history of
22q11.2DS pertains primarily to neuropsychiatric, endocrine, cardiovascular, reproductive, and psychosocial issues.

Although a growing number of adults with 22q11.2DS received their diagnosis during childhood because of targeted testing prompted by typical syndromic features, the variable phenotype of 22q11.2DS often presents a significant diagnostic challenge to clinicians. Many of the adults reported in the literature and most familiar to geneticists were diagnosed following the birth of an affected child. ${ }^{6}$ Others were reported as a result of genetic screening studies of at-risk populations. ${ }^{5}$ Multiple case reports in subspecialty journals testify to the limited awareness in the general medical community of 22q11.2DS and its vast phenotypic spectrum. For this reason, many adults with a 22q11.2 deletion, especially those without typical congenital anomalies or who were born before confirmatory testing became available, remain undiagnosed. ${ }^{7}$ This is the greatest barrier to understanding the full spectrum of the condition, including course and outcome, and thus to providing the optimal anticipatory care that promises improved symptom management, quality of life, and functioning. The available data support the likelihood that all associated conditions in 22q11.2DS respond similarly to the idiopathic forms of these conditions, that is, to standard management strategies and treatments, whether surgical or medical. The caveat for 22q11.2DS is that the multisystem nature of the associated features and potential side effects of treatment demand attention by all clinicians, regardless of their subspecialty. These issues are outlined in Tables 1-4 and Supplementary Figure S1 online.

\section{Neuropsychiatric manifestations in young to middle-aged adults}

Neuropsychiatric diseases (i) comprise the most common group of later-onset conditions in 22q11.2DS, ${ }^{8-13}$ (ii) are typically of greatest concern to patients and their families because of their seriousness and the associated stigma, ${ }^{14,15}$ (iii) are most likely to bring adolescent and adult patients (back) to medical attention and to affect the individual's daily functioning, ${ }^{15,16}$ and (iv) may constitute a management challenge. ${ }^{11,17,18}$

\section{Schizophrenia}

There is a well-established association between 22q11.2DS and schizophrenia. ${ }^{19,20}$ Approximately one in every four to five adults with 22q11.2DS will develop this serious mental illness, usually in late adolescence or early adulthood. ${ }^{8,10}$ Thus individuals with 22q11.2DS have a greater than 20 -fold increase in risk for schizophrenia. ${ }^{10,19}$ Schizophrenia associated with 22q11.2DS is essentially indistinguishable from schizophrenia in the general population with respect to prodrome, age at onset, presentation, and cognitive profile, apart from a lower mean intelligence quotient. ${ }^{8,10,19,21-25}$ As for virtually all associated conditions in 22q11.2DS, standard management is recommended, that is, management according to clinical practice guidelines for schizophrenia, including antipsychotic medications. ${ }^{1}$ Patients may benefit from a "start low, go slow" approach to antipsychotic dosing and prophylactic (e.g., anticonvulsant) 
Table 1 Recommendations for periodic assessments and health monitoring for adults with 22q11.2DS

\begin{tabular}{|c|c|c|c|c|}
\hline Recommendations & \multicolumn{2}{|c|}{$\begin{array}{l}\text { Baseline workup at adult diagnosis or initial } \\
\text { assessment at transition from pediatric care }\end{array}$} & \multicolumn{2}{|c|}{$\begin{array}{l}\text { Annual or biennial follow-up } \\
\text { as an adult }\end{array}$} \\
\hline \multicolumn{5}{|l|}{ Genetic/general assessments and management } \\
\hline Genetic counseling $^{a}$ & $\checkmark$ & & $\checkmark$ & \\
\hline \multicolumn{5}{|l|}{ Other clinical assessments } \\
\hline Comprehensive medical history & $\checkmark$ & & & \\
\hline Systems review & $\checkmark$ & & $\checkmark$ & \\
\hline Psychiatric assessment ${ }^{c}$ & $\checkmark$ & & & $\checkmark$ \\
\hline Cognitive and capacity assessments & $\checkmark$ & & & $\checkmark$ \\
\hline Family history & $\checkmark$ & & $\checkmark$ & \\
\hline Physical examination (including for hygiene and care) & $\checkmark$ & & $\checkmark$ & \\
\hline $\mathrm{BMI} /$ growth/nutritional assessment & $\checkmark$ & & $\checkmark$ & \\
\hline \multicolumn{5}{|l|}{ Other investigations } \\
\hline 22q11.2DS-relevant laboratory tests ${ }^{d}$ & $\checkmark$ & & $\checkmark$ & \\
\hline Electrocardiography & & $\checkmark$ & & $\mathfrak{S}^{\mathrm{e}}$ \\
\hline Transthoracic echocardiography & & $\begin{array}{l}\checkmark \text { (If not previously } \\
\text { performed) }\end{array}$ & & $\boldsymbol{J}^{\mathrm{e}}$ \\
\hline Abdominal ultrasound ${ }^{f}$ & & $\checkmark$ & & $\checkmark$ \\
\hline Electroencephalography & & $\checkmark$ & & $\checkmark$ \\
\hline
\end{tabular}

22q11.2DS, 22q11.2 deletion syndrome; BMI, body mass index.

aDescribed in the text. 'Proband, offspring, and parents (siblings if parents unavailable). In addition to monitoring for changes (Table 2), includes assessment of, e.g., substance use, gambling, and risky behaviors. ${ }^{\mathrm{C} C} \mathrm{~m}$ plete blood count (CBC) and differential, electrolytes, thyroid-stimulating hormone, $\mathrm{pH}$-corrected ionized calcium, magnesium, parathyroid hormone, creatinine, liver function tests (especially alanine aminotransferase), lipid profile, glucose, and HbA1c are examples. Consider checking

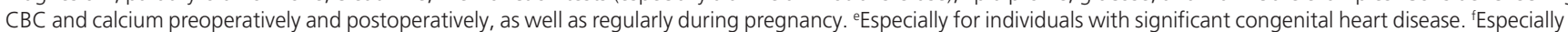
with regard to renal agenesis. Including vocational counseling/training, supported employment and individual placement and support, diet and exercise counseling, life skills assessment, and financial management.

Adapted from refs. 1, 9, and 16 .

management strategies to help ameliorate the risk of associated side effects, particularly with respect to seizures during clozapine treatment. ${ }^{26}$

The elevated risk for psychotic illness in 22q11.2DS prompts questions about prevention, early signs, diagnosis, and treatment. Informed discussion about schizophrenia as a lifelong but treatable and manageable condition is essential. Placing the disease in the context of other chronic diseases such as diabetes mellitus may be helpful. Promptly seeking expert help in diagnosis and effective treatment may improve prognosis. ${ }^{19}$ Learning about early signs that may herald treatable psychiatric illness can facilitate this and may be empowering for families
(Table 2). There are no proven preventions for psychotic illness. However, avoiding substance use, particularly early marijuana use, and implementing lifelong general health measures such as good nutrition and physical and mental exercise are reasonable recommendations. ${ }^{27}$ Research with respect to identifying predictors of future psychotic illness in 22q11.2DS is ongoing. ${ }^{25,28-30}$ Meanwhile, concerns, misconceptions, and a sense of stigma should be solicited and addressed..$^{14,15,31}$

\section{Other psychiatric disorders and neurobehavioral features}

Other nonpsychotic, treatable psychiatric illnesses are collectively more common in 22q11.2DS than is schizophrenia, and 
Table 2 Signs and symptoms representing a change from baseline that may suggest a treatable psychiatric illness New onset or exacerbation of problems

\section{Thinking}

- Impaired memory, concentration, or attention

- Preoccupations

- Increased irrational statements or repetitive ideas

- Misinterpretation of people's motives, situations

- Suspiciousness

- Threatening suicide

- Delusions and hallucinations (changed perception of reality, e.g., believe phone is tapped, hearing voices, new onset of imaginary friends)

\section{Behavior}

- Avoidance of people, social withdrawal (even from family)

- Increased impulsive behaviors and/or emotional outbursts

- Agitation (e.g., screaming, pacing, aggression)

- Unusual/odd or self-injurious behavior

- Neglect in self-care (e.g., hygiene, clothing, appearance)

- Deterioration in functioning at home, in social situations, at school or work

\section{Emotions}

- Increased anxiety, worry, nervousness, fear

- Irritability, anger, hostility, resentment

- Increased sadness, crying

- Increased apathy, not as interested in or enjoying life

- Smiling or laughing for no apparent reason

- Rapidly changing mood-from happy to sad to angry for no apparent reason

- Hypersensitivity to perceived criticisms/insults (hurt feelings)

\section{Physical/somatic}

- Changes in amount of sleep (much less or much more)

- Disruption of sleep patterns

- Changes in energy level (e.g., increased fatigue)

- Changes in appetite and/or weight

- Increased motor disturbances (e.g., tremors, tics)

- Increase in physical complaints (e.g., gastrointestinal symptoms)

\section{Differential diagnosis/potential confounding or exacerbating factors}

- Endocrine (e.g., thyroid dysregulation, hypocalcemia) or other processes (e.g., infection, sleep apnea, drinking excess water/carbonated beverages, Parkinson disease, emerging dementia) causing, e.g., metabolic disturbance or hypoxia

- Substance use (e.g., caffeine, alcohol, street drugs such as marijuana)

- Treatment related (medication side effects or undertreatment, e.g., secondary to poor/improper compliance)

- Changes in physical environment (e.g., caregivers, co-residents, living space)

- Hearing or other sensory deficits

Adapted from refs. 19 and 27

the incidence of some may be higher even than the high rates of these conditions in the general population..$^{8-10,13}$ Anxiety disorders seem to be particularly common in adults with 22q11.2DS, sometimes persisting from childhood and sometimes arising later. ${ }^{10}$ Notably, although bipolar disorder sometimes occurs in $22 \mathrm{q} 11.2 \mathrm{DS}$, it has a similar prevalence to that in the general population, as do other more common disorders such as major depression and substance use disorders. ${ }^{8,9,13,32}$ Autism spectrum disorders and sometimes attention deficit disorder, with onset during childhood, remain important features in adulthood ${ }^{13,33}$ but have no apparent relationship to the later appearance of schizophrenia. ${ }^{33}$

With respect to the diagnosis and treatment of psychiatric disorders, challenges exist in the minority of adults with moderate or severe intellectual disability, ${ }^{12,17}$ including communication limitations that may hinder the recognition or assessment of treatment response using standard measures. In most cases these challenges can be overcome with expert care. Emotional or temper outbursts, which are described in individuals with 22q11.2DS, may be a feature of untreated or undertreated anxiety or psychotic illness, and multiple physical factors potentially contribute to them. ${ }^{1,19}$ Early diagnosis and prompt institution of standard effective management by a knowledgeable clinician are essential for all psychiatric illnesses. Routine monitoring for changes in emotions, thinking, physical state, and behavior/ functioning (Table 2) facilitates this. Awareness of issues such as suggestibility, the patient's intellectual level, and, most important, his or her baseline state and functioning, is essential.

\section{Cognitive and adaptive functioning}

Most patients with 22q11.2DS have an intelligence quotient in the borderline range (70-84), and $30-40 \%$ have mild intellectual disability (intelligence quotient: 55-69). ${ }^{21,23,28,34}$ More severe intellectual disability is rare, ${ }^{9,23,35}$ but 22q11.2DS may be underrecognized in this subgroup. ${ }^{12} \mathrm{~A}$ minority of individuals have intellect in the average range, although various learning difficulties may still be present, for example, in arithmetic skills. ${ }^{9,23,36}$ These may require accommodations in postsecondary education and workplace settings. Most affected individuals require assistance with understanding and completing forms (e.g., to ensure benefits are received), managing money, and making complex life and work decisions. Structure and routine usually facilitate optimal functioning in all domains and can help reduce anxiety. Using visual reminders may help overcome deficits in verbal (auditory) learning and reduce frustrations for caregivers and patients alike.

On the other hand, deficits in receptive language and comprehension may be camouflaged by relatively good verbal skills, despite speech deficits. Coupled with a tendency to downplay, hide, and/or deny existing problems, as well as difficulty engaging 
Table 3 General recommendations for prenatal and perinatal care of adults with 22q11.2DS

\section{General recommendation}

Discuss pregnancy options

Ensure provision of genetic counseling

Optimize psychiatric care for mother and fetus, as well as partner, if necessary

Genetic diagnosis of 22q11.2 deletion in fetus/conceptus

Evaluate for potential fetal anomalies

Provide and reinforce general recommendations

Consider delivery at a tertiary care center and/or a center experienced in the care of neonates with 22q11.2DS and associated obstetric complications

\section{Details}

- With both partners, as well as family members and health professionals, as appropriate

- Including both partners, as well as other guardians/caregivers, as appropriate

- Discussion of (i) recurrence risk (50\% chance of having a child with 22q11.2DS), (ii) unpredictable intrafamilial variability in expression, (iii) potential impact of maternal morbidities and associated treatments on maternal-fetal health, and (iv) challenges in caring for a child with (or without) 22q11.2DS

- Monitor for and promptly treat gestational diabetes

- Regular 22q11.2DS laboratory investigations, e.g., TSH, ionized calcium (Table 1)

- Calcium and vitamin D supplementation

- Confirm medication safety (e.g., at http://www.motherrisk.org) and necessity, and modify medications only as required

- Monitor for signs of peri- and postpartum mood and psychotic disorders

- Standard prenatal testing using FISH, MLPA, or chromosomal microarray to analyze chorionic villus sample (beginning at 10-12 weeks' gestation) or chromosome preparations from fetal cells obtained by amniocentesis (beginning at 15-18 weeks' gestation)

- Preimplantation genetic diagnosis and assisted reproductive techniques would be available in some jurisdictions for select cases

- Noninvasive prenatal genetic testing is an emerging technology

- High-resolution ultrasound examination for polyhydramnios, intrauterine growth retardation and palatal, renal, and other anomalies (beginning at 18-22 weeks' gestation)

- Fetal echocardiogram (18-22 weeks' gestation)

- Maintain good nutrition and physical activity

- Standard preconception folate/vitamin supplementation

- Avoid smoking, alcohol, and street drugs

- Avoid known teratogens (e.g., retinoic acid)

- Women with significant congenital cardiac conditions should also be monitored by their cardiologists

22q11.2DS, 22q11.2 deletion syndrome; FISH, fluorescence in situ hybridization; MLPA, multiplex ligation-dependent probe amplification; TSH, thyroid-stimulating hormone.

Adapted from refs. 19 and 70

during a first meeting, medical history taking may be challenging. Patience, along with collateral information from caregivers who know the patient well, can ameliorate these issues. However, more time and more investigations than are necessary for the average patient are often required. Similarly, the cognitive capability of patients with 22q11.2DS to describe or to understand the various medical conditions they have and the need for treatment for these conditions may be suboptimal, especially in the presence of intellectual disability and/or psychotic illness. ${ }^{16,23}$ Standard measures to improve compliance with recommended management can include clear and careful explanations to the patient-together with caregivers-about their conditions and treatments. Providing simple, written instructions and monitoring medication intake are often helpful.

Cognitive impairments may have an impact on many domains of functioning, including communication, living skills (particularly in work settings), and management of finances. ${ }^{16,23}$ The presence of a serious psychiatric disorder such as schizophrenia can also be an important mediator of functioning. ${ }^{16,23}$ For many patients, the availability of supported employment opportunities and close collaboration between the vocational team (employment specialists) and the clinical team can facilitate finding an appropriate job and job retention. Part-time employment may be preferred and/or more accommodations provided (e.g., more breaks), especially with limitations such as fatigue and stress sensitivity that may accompany medical or neuropsychiatric conditions. Areas of relative strength in adult functioning and data that could help to inform vocational training and expectations are described in more detail elsewhere. ${ }^{16}$

Deficits in socialization, comprehension, and executive functioning ${ }^{16,21,23,37}$ can make some individuals with 22q11.2DS, even those with intellect in the average range, prone to poor social judgment and decision making in everyday life. They may become involved in financially and/or emotionally exploitative friendships or romantic relationships without realizing their abusive nature. Internet safety has also become a major issue (e.g., sharing of intimate photos or other personal information). Lack of insight into limitations may lead to setting unrealistic goals. Balancing the individual's wishes and aspirations with attempting to avoid repeated disappointments that are highly discouraging may be a challenge. Family members and professionals involved in the care of patients with 22q11.2DS should be cognizant of these potential problems in order to provide support accordingly. 
Table 4 Genetic counseling strategies for adults with 22q11.2DS with intellectual disabilities and/or psychotic illness

\section{Traditional genetic counseling approach}

Assumption of average intelligence

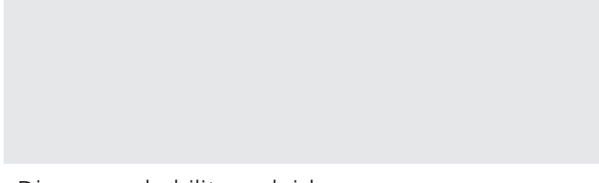

Discuss probability and risk

Minimal use of visual aids

Use analogies to illustrate concepts

A single genetic counseling session

Nondirectiveness

Expects to be told what to do

Identify patient's past decision-making strategies

Openly discuss psychosocial issues (e.g., abilities, reaction to stressful situations and level of responsibility, fears and concerns, suggestibility)

Confidentiality, patient autonomy

Functions as part of a "system" that often

Use patient letter and literature to supplement counseling includes family and professionals

Limited reading abilities

Pervasive but extremely variable level of learning difficulties and cognitive impairment

Difficulty understanding abstract concepts

Visual memory may be a relative strength compared with auditory memory

Difficulty generalizing from one situation to another

Attentional dysfunction and other cognitive impairments

Little or no experience in complex decision making

Denial of learning or social problems

rimited reading abilities

Alternative genetic counseling strategy for patients with 22q11.2DS

Spend time gaining an understanding of the specific level of cognitive and practical functioning, as well as any specific learning disabilities

Obtain neurocognitive testing or refer to existing test results

Minimize discussion of probabilities and risk

Use simple diagrams and visual aids to illustrate all major points Provide handouts

Minimize use of analogies to illustrate concepts

Ensure frequent repetition and reinforcement of information over the course of multiple genetic counseling sessions

Provide a written summary to patient and caregivers

Based on discussion of patient's feelings and attitudes, summarize, rephrase, and verify patient's decision

With patient's permission, involve his/her support system in making appointments, giving reminders, and reviewing/reinforcing information discussed during genetic counseling

Elicit patient's feelings and attitudes related to the current decisions

Use nonjudgmental language to elicit history Use patient's preferred term for his/her own disability, even if not technically correct

Recognize that many genetic counseling tasks may be accomplished with involvement of the patient's support system

With permission, engage a support person to review summary letter with patient Use visual diagrams/handouts

22q11.2DS, 22q11.2 deletion syndrome.

Adapted from ref. 74

\section{Financial and other competency-related issues}

Legally, individuals are generally presumed to be competent to make financial, medical, and personal decisions when they reach the age of majority. Adults with a low baseline intellectual level, specific cognitive deficits, and/or psychotic illness, however, may have compromised competencies and, in particular, increased vulnerability to exploitation. With weakness in arithmetic skills common in 22q11.2DS, ${ }^{23,36}$ financial competency may need to be assessed in early adulthood and followed up; then, steps to manage identified weaknesses in money management and/or to determine formal financial incompetency should be taken. Extra lessons and guidance in shopping and budgeting, as well as supervised use of bank accounts among patients with weak financial skills, may be beneficial. In some cases, powers of attorney or legal guardianships may be needed to legally allow a competent parent or caretaker to manage an adult patient's financial matters.

As for anyone, it would be prudent for patients with 22q11.2DS and their family members to discuss their medical wishes and designate a substitute decision maker or establish a power of attorney or an advance medical directive in preparation for any future development of incompetence because of accident, physical illness, and/or psychiatric illness.

\section{Seizures}

Single and recurrent seizures are common across the life span and may be unprovoked or related to identifiable factors, especially hypocalcemia. ${ }^{9,38}$ The lifetime prevalence of epilepsy in patients with 22q11.2DS is in the range of 5 to $7 \%,{ }^{9,38}$ far greater than in the general population $(0.5-1.0 \%)$. There are 
limited data about the type, localization, and cause of seizures in 22q11.2DS, particularly in adults. ${ }^{39}$ Precipitating events can include fever, ischemia, hypoxia, surgery, medications (including antipsychotics), ${ }^{26}$ hyperprolinemia, and hypocalcemia. Hypocalcemia can trigger seizures at any age, ${ }^{40,41}$ even in patients with no history of hypocalcemia and/or seizures, because of the underlying parathyroid dysfunction that is part of the syndrome. Hypocalcemic seizures generally resolve with appropriate supplementation ${ }^{1}$ and monitoring alone, but anticonvulsant therapy may be indicated should seizures continue after ionized calcium concentrations have normalized. ${ }^{42}$

Some patients have generalized tonic-clonic seizures, with or without myoclonic jerks, characterized as generalized epilepsy. Other seizures may present with minor symptoms such as brief episodes of behavioral arrest and loss of consciousness with or without automatisms. This form of dyscognitive (or complex partial) seizure may be interpreted as confusion or memory loss, potentially leading to underestimates of the true prevalence of seizures in 22q11.2DS. Electroencephalography and computed tomography and/or magnetic resonance imaging of the brain are indicated for all adults with 22q11.2DS with a history suggesting potential nonhypocalcemic seizures. In some cases, seizures may be related to cortical malformation such as polymicrogyria, periventricular nodular heterotopia, or cortical dysplasia. ${ }^{39,40}$

Published case reports indicate that response to standard treatment with anticonvulsant medications seems typical, ${ }^{38,42}$ but as yet there are limited data. Standard cautions with respect to the need for vitamin supplements with many anticonvulsants would apply.

\section{Endocrine issues}

Hypocalcemia (either overt or subclinical/latent) occurs in the majority of patients with 22q11.2DS and may arise at any age, especially after puberty. ${ }^{1,9,40,43-46}$ The largest study of adults reported $80 \%$ with a lifetime history of hypocalcemia, including evidence that for most cases of neonatal hypocalcemia, the hypocalcemia recurred later in life. ${ }^{46}$ Hypocalcemia in 22q11.2DS is typically attributable to hypoparathyroidism. ${ }^{44,47}$ Recent data also suggest that in some cases hypothyroidism and hypomagnesemia may be associated findings. ${ }^{41}$ Hypocalcemia can be associated with fatigue, emotional irritability, abnormal involuntary movements of any sort, seizures, and cardiac arrhythmias (electrocardiographic changes including prolongation of the QT interval) and may predispose to osteopenia/ osteoporosis. There is an increased risk of hypocalcemia with any biological stress, such as surgery, childbirth, or infection. Hypocalcemia may also be worsened by alcohol or carbonated beverages such as colas.

Regular investigations including measurements of $\mathrm{pH}$-corrected ionized calcium, magnesium, parathyroid hormone, and creatinine concentrations are recommended. ${ }^{1}$ Daily calcium and vitamin $\mathrm{D}$ supplementation are recommended for all adults with 22q11.2DS. ${ }^{1,9}$ Magnesium supplementation for those with hypomagnesemia is indicated. ${ }^{41}$ Treatment with hormonally active metabolites of vitamin D for more severe hypocalcemia usually requires consultation with an endocrinologist. Targeted monitoring of calcium concentrations should be considered at vulnerable times, for example, peri-operatively, perinatally, or during severe illness. Caution is advised with respect to overcorrection, which can result in iatrogenic hypercalcemia, renal calculi, and renal failure. This can occur inadvertently, such as when treatment compliance with calcitriol improves after adequate management of a psychiatric illness.

Routine clinical monitoring for autoimmune thyroid disease is also indicated. ${ }^{9,41,48}$ More than 1 in 4 adults may develop hypothyroidism, ${ }^{41}$ and 1 in 20 has hyperthyroidism. ${ }^{9}$ As for hypocalcemia, symptoms of thyroid disease may be confused with those of psychiatric and other conditions. ${ }^{41}$ Thyroid function should be assessed on an annual basis. ${ }^{1}$ Standard treatments seem effective. ${ }^{41}$

Obesity has been reported in up to $35 \%$ of individuals affected with 22q11.2DS, ${ }^{9}$ often with onset during childhood or the teenage years ${ }^{49}$ and sometimes with rapid weight gain. Obesity and antipsychotic medication use are known predisposing factors for the development of type 2 diabetes mellitus, metabolic syndrome, and nonalcoholic fatty liver, the prevalences of which in 22q11.2DS are as yet unknown. Standard dietary and exercise measures are recommended, taking into account the other associated neuropsychiatric and medical conditions present.

\section{Immune deficits}

The severity and prevalence of immune deficits present in 22q11.2DS are highly variable, although complete thymic aplasia is rare. ${ }^{50}$ The immunocompromise found in children sometimes persists into adulthood, including failure to mount an antibody response to vaccination. ${ }^{51}$ In general, however, autoimmune disorders are the major immune system issues in adults. ${ }^{1}$ These represent the full range of possibilities, including the endocrine disorders mentioned above; others, such as adult arthritic conditions, await formal study.

\section{Cardiovascular manifestations}

Congenital cardiac anomalies are common in patients diagnosed with 22q11.2DS as children ${ }^{2}$ and represent a chronic disease that requires regular follow-up during adulthood. Although early studies reported serious congenital heart disease to be present in most patients with $22 \mathrm{q} 11.2 \mathrm{DS},{ }^{40,52}$ if ascertainment outside of cardiac clinics is taken into account, the prevalence seems to be about $40 \%{ }^{9.53}$ Echocardiography during adolescence or adulthood may also reveal aortic root dilation or other minor cardiac anomalies in small numbers of patients. ${ }^{54}$

The most common serious congenital cardiac lesions requiring ongoing care in adults with 22q11.2DS are conotruncal defects, especially tetralogy of Fallot. In developed countries adults with tetralogy of Fallot have almost always undergone intracardiac repair during childhood. A significant proportion of these adults require repeat cardiac interventions, such as pulmonary valve replacement for severe residual pulmonic 
regurgitation. ${ }^{55,56}$ Cardiac determinants of outcome include the presence of residual valve lesions (regurgitation or residual obstruction), ${ }^{57}$ the systolic function of the right and left heart, ${ }^{58}$ and the propensity of the patient to arrhythmias. ${ }^{57}$ Morphological variants such as pulmonary atresia, doubleoutlet right ventricle, atrioventricular septal defect, and branch pulmonary artery stenosis are important factors in adverse late outcomes ${ }^{59}$ and these are more prevalent in patients with 22q11.2DS than in other patients. Management strategies are lesion specific and are discussed in detail elsewhere. ${ }^{55,56}$ Other congenital cardiovascular conditions associated with 22q11.2DS include other conotruncal defects (e.g., interrupted aortic arch), as well as pulmonary stenosis, septal lesions (e.g., ventricular septal defect, patent ductus arteriosus), vascular rings, left-sided lesions, and other aortic arch abnormalities. Ongoing follow-up may be warranted. ${ }^{55,56}$

\section{Sexual health and reproductive issues in 22q11.2DS}

Although reproductive fitness is reduced, ${ }^{60}$ romantic partnerships, sexual activity, and pregnancy are important aspects of adult life for many individuals with 22q11.2DS. Developmentally appropriate sex education and routine reproductive health care (including contraceptive counseling for men and women, and Pap tests and sexually transmitted infection screening, as appropriate) are indicated.

General recommendations for managing pregnancies for which one member of the couple has 22q11.2DS are provided in Table 3. Many common manifestations of 22q11.2DS, including endocrine, cardiac, and psychiatric disease, can pose risks to the mother and fetus during pregnancy and the postpartum period. In an unpublished case series of pregnancies of women with 22q11.2DS, spontaneous fetal loss (miscarriage/stillbirth), pregnancy complications, prematurity, and low birth weight were common (A.S.B., unpublished data). There is evidence of elevated risks for fetal/neonatal complications in pregnancies in which the fetus has a 22q11.2 deletion, ${ }^{61,62}$ warranting consideration of high-risk antepartum care. Specific pregnancy and contraception education is required for women with congenital cardiac lesions because pregnancies in this group are associated with increased risks of maternal and fetal/neonatal complications. ${ }^{56,63}$

In some individuals delayed maturity and poor decisionmaking abilities may increase the likelihood of engaging in high-risk sexual practices. ${ }^{60}$ Resources and guidelines for sexuality and relationship education for adults with intellectual disabilities or other genetic syndromes such as trisomy 21 may be helpful when counseling individuals with 22q11.2DS. Notably, women with 22q11.2DS who have neither schizophrenia nor intellectual disability, regardless of the presence of cardiac anomalies, may have a similar number of offspring as their unaffected sisters, i.e., near normal reproductive fitness. ${ }^{60}$

\section{Outcomes in older adults}

Information about long-term outcome and older age ranges in 22q11.2DS is limited; most reports involve individuals in their mid-30s, on average. The substantial proportion of patients living into middle adulthood presents a new set of challenges for long-term care. The aging parents of the current cohort of adults with 22q11.2DS are often faced with the complexities of managing their adult children in their home or elsewhere, which may present significant financial, physical, and emotional stress. A close partnership between families and appropriate professional agencies, including social work and occupational therapy, can help address these challenges. Spouses, siblings, and other relatives may also play an important role. ${ }^{6,15,64}$

\section{Early-onset Parkinson disease and neurodegenerative diseases}

There is accumulating evidence that $22 \mathrm{q} 11.2 \mathrm{DS}$ is associated with an increased risk for early-onset (age $<50$ years) Parkinson disease. ${ }^{65-67}$ In one study the occurrence of Parkinson disease was significantly elevated over population expectations: $5.9 \%$ of 68 patients aged 36 to 64 years were diagnosed with Parkinson disease. ${ }^{67}$ All showed early onset of motor symptoms and typical symptom pattern, disease course, and treatment response. Periodic neurological assessment for signs of parkinsonism should thus be considered, especially in adults presenting with changes in motor functioning. Atypical antipsychotics that are associated with fewer extrapyramidal side effects-for example, clozapine and quetiapine-are likely to be preferable for patients who have both Parkinson disease and schizophrenia. ${ }^{67}$ Calcium concentrations should be monitored appropriately because hypocalcemia may induce or aggravate existing tremors. ${ }^{67}$ Functional imaging, where available, may help to distinguish Parkinson disease from extrapyramidal side effects of antipsychotics. ${ }^{66,67}$ There are as yet few studies of other neurodegenerative disorders in 22q11.2DS. Individuals with severe intellectual disability and psychotic illness may develop a form of dementia. ${ }^{12}$

\section{Premature mortality in 22q11.2DS}

Individuals with 22q11.2DS who survive childhood seem to be at increased risk of premature mortality as adults, but this mortality is not attributable to any single identifiable factor. In one study of 102 adults with 22q11.2DS (mean age: 33.6 years), survival rates to ages 40 and 50 years were 89.9 and $73.9 \%$, respectively. ${ }^{68}$ The median age of death among 12 individuals was 41.5 years (range: 18.1-68.6 years), and the causes of death reported were sudden death (most common), heart failure, stroke, suicide, and postoperative complications ${ }^{68}$ Further studies including essential postmortem data are required to better define life expectancy, the mechanism responsible for sudden death, and the roles of major associated conditions.

\section{Genetic counseling for adults with 22q11.2DS}

Genetic counseling is an essential component in the ongoing management of all patients with 22q11.2DS and concerned relatives at multiple time points, from late adolescence through adulthood. ${ }^{1}$ Follow-up genetic counseling may also be necessary as knowledge about 22q11.2DS continues to expand. Although 
genetic counseling would ideally be provided by a trained genetic counselor or medical geneticist, all clinicians involved in the ongoing care of an adult with 22q11.2DS should be in a position to reinforce the information provided in genetic counseling sessions, particularly as it pertains to their medical specialty.

For adults who were diagnosed during childhood, a best-case scenario would mean early interventions for developmental delay and learning difficulties, ${ }^{69}$ anticipatory medical care that may reduce morbidity, ${ }^{9,19,41,70}$ and genetic counseling that provides updated information about the basic genetics and lateronset features. However, the extent of genetic counseling varies depending on the age, location, and presentation of the individual at diagnosis and follow-up, as well as the background of the clinician providing counseling. Children may not be included in, or may not understand, the genetic counseling provided. Furthermore, adolescents transitioning out of pediatric care often do not yet possess the maturity, curiosity, or educational preparation to fully appreciate the content and message of genetic counseling. Individuals diagnosed following the birth of their child with 22q11.2DS may be more likely to have a milder clinical presentation, ${ }^{6,7,60,71,72}$ but genetic counseling and a full systems review and standard investigations are indicated and may reveal previously undetected medical conditions (e.g., hypocalcemia, renal abnormalities). ${ }^{2,73}$

Given the advantages of early detection and treatment of lateonset features of 22q11.2DS, all adolescents and adults should be informed of known clinical manifestations of the syndrome. There may previously have been concerns about "information overload" for the patient or apprehension about fully discussing the wide spectrum of possible phenotypes, especially the increased risk for psychotic illness. ${ }^{14,31} \mathrm{~A}$ matter-of-fact approach with a competent and knowledgeable professional will help in all situations. Genetic counseling for adolescents and young adults especially should include discussion of, and preparation for, transition issues and reproductive decision making.

Basic genetic counseling includes the fact that an affected individual, male or female, has a $50 \%$ chance of having an affected child at each pregnancy. In light of the variability of the syndrome, predicting the range and severity of manifestations in the offspring is impossible. Explaining to adults with 22q11.2DS how their child may be "like them" (in having a 22q11.2 deletion) and yet not "like them" (in having a different phenotype) can be challenging. A diagram showing the contribution of a different intact chromosome 22 for a parent and offspring may be helpful (Supplementary Figure S2 online). Notably, a parent who has experienced a life with 22q11.2DS may have a different perception of what the condition is compared with an unaffected couple discovered to have an affected fetus or infant. ${ }^{53}$ When an adult is identified as having 22q11.2DS only after the birth of an affected child, the parent requires genetic counseling that focuses on his or her own diagnosis. This often includes the need for additional support in managing the care of the affected child. ${ }^{6,53,70}$

Traditional genetic counseling approaches must be modified to take into account the learning disabilities and other neuropsychiatric issues that are common in adults with 22q11.2DS ${ }^{1,23,74}$ (Table 4). Finucane ${ }^{74}$ presented sample dialogue from a genetic counseling session involving an adult with 22q11.2DS and intellectual disability to illustrate her technique. In our experience most adults with 22q11.2DS are able to learn simple facts about the genetics of their condition and are satisfied with the standard genetic counseling process, provided there are the necessary modifications to traditional techniques.

\section{CONCLUSIONS}

Widespread use of genome-wide genetic diagnostic technologies, declining infant mortality with advances in pediatric surgery and care, nonzero reproductive fitness, and a 50\% recurrence risk in 22q11.2DS predict a growing population of (diagnosed and undiagnosed) adults with 22q11.2DS. ${ }^{60,70}$ Increasing detection of 22q11.2DS is expected to place greater demands on health-care providers, in particular with the introduction of noninvasive prenatal testing for microdeletion syndromes including 22q11.2DS. Studies are needed to assess impact on health-care resources, given the likely high associated healthcare costs and use of medical services by adults with 22q11.2DS.

More information about adult outcomes in 22q11.2DS is urgently needed to assist accurate genetic counseling and to improve care and service planning. Many important conditions in the general adult population (e.g., cancer, chronic autoimmune conditions, and hematologic issues) are understudied in 22q11.2DS. There is little evidence to support 22q11.2DS-specific management for associated conditions, and as yet there are few dedicated adult 22q11.2DS clinics. The issues identified herein with respect to 22q11.2DS may be common to multisystem genetic syndromes in adulthood more generally, and will increasingly challenge the traditional pediatric focus of clinical genetics services.

\section{SUPPLEMENTARY MATERIAL}

Supplementary material is linked to the online version of the paper at http://www.nature.com/gim

\section{ACKNOWLEDGMENTS}

The authors acknowledge the support and endorsement provided by The 22q11.2 Society (http://www.22qsociety.org) on this work, with special thanks to its president, Peter Scambler, for his helpful comments on the manuscript. They also thank Benjamin Gould and Alina Guna for assistance with the literature search and Monica Torsan for assistance with manuscript formatting. This work was supported by the Canadian Institutes of Health Research (CIHR) (MOP 97800, MOP 111238) and Canada Research Chairs program (A.S.B.), a Dempster Family Foundation Canada grant (W.L.A.F. and A.S.B.), a CIHR Frederick Banting and Charles Best Graduate Scholarship and Brain Canada Mental Health Training Award (N.J.B.), a CIHR Vanier Canada Graduate Scholarship and a McLaughlin Centre MD/PhD studentship (G.C.), and a Dalglish Family Hearts and Minds Clinic Fellowship (E.B.). A.S.B. holds the Dalglish Chair in 22q11.2 Deletion Syndrome. 


\section{DISCLOSURE}

The authors declare no conflict of interest.

\section{REFERENCES}

1. Bassett AS, McDonald-McGinn DM, Devriendt K, et al.; International 22q11.2 Deletion Syndrome Consortium. Practical guidelines for managing patients with 22q11.2 deletion syndrome. J Pediatr 2011;159:332-339.e1.

2. McDonald-McGinn DM, Sullivan KE. Chromosome 22q11.2 deletion syndrome (DiGeorge syndrome/velocardiofacial syndrome). Medicine (Baltimore) 2011:90:1-18.

3. Costain G, McDonald-McGinn DM, Bassett AS. Prenatal genetic testing with chromosomal microarray analysis identifies major risk variants for schizophrenia and other later-onset disorders. Am J Psychiatry 2013;170:1498.

4. Kaminsky EB, Kaul V, Paschall J, et al. An evidence-based approach to establish the functional and clinical significance of copy number variants in intellectual and developmental disabilities. Genet Med 2011;13:777-784.

5. Cooper GM, Coe BP, Girirajan S, et al. A copy number variation morbidity map of developmental delay. Nat Genet 2011;43:838-846.

6. McDonald-McGinn DM, Tonnesen MK, Laufer-Cahana A, et al. Phenotype of the 22q11.2 deletion in individuals identified through an affected relative: cast a wide FISHing net! Genet Med 2001;3:23-29.

7. Vogels A, Schevenels S, Cayenberghs R, et al. Presenting symptoms in adults with the 22q11 deletion syndrome. Eur J Med Genet 2014;57:157-162.

8. Murphy KC, Jones LA, Owen MJ. High rates of schizophrenia in adults with velocardio-facial syndrome. Arch Gen Psychiatry 1999;56:940-945.

9. Bassett AS, Chow EW, Husted J, et al. Clinical features of 78 adults with 22 q11 seletion syndrome. Am J Med Genet A 2005;138:307-313.

10. Fung WLA, McEvilly R, Fong J, Silversides C, Chow E, Bassett A. Elevated prevalence of generalized anxiety disorder in adults with 22q11.2 deletion syndrome. Am J Psychiatry 2010;167:998

11. Philip N, Bassett A. Cognitive, behavioural and psychiatric phenotype in 22q11.2 deletion syndrome. Behav Genet 2011:41:403-412.

12. Evers LJ, van Amelsvoort TA, Candel MJ, Boer H, Engelen JJ, Curfs LM Psychopathology in adults with 22q11 deletion syndrome and moderate and severe intellectual disability. J Intellect Disabil Res 2014:58:915-925.

13. Schneider $M$, Debbané $M$, Bassett $A S$, et al.; International Consortium on Brain and Behavior in 22q11.2 Deletion Syndrome. Psychiatric disorders from childhood to adulthood in 22q11.2 deletion syndrome: results from the International Consortium on Brain and Behavior in 22q11.2 Deletion Syndrome. Am J Psychiatry 2014;171:627-639.

14. Hercher L, Bruenner G. Living with a child at risk for psychotic illness: the experience of parents coping with 22q11 deletion syndrome: an exploratory study. Am J Med Genet A 2008:146A:2355-2360

15. Karas DJ, Costain G, Chow EW, Bassett AS. Perceived burden and neuropsychiatric morbidities in adults with $22 q 11.2$ deletion syndrome. J Intellect Disabil Res 2014:58:198-210.

16. Butcher NJ, Chow EW, Costain G, Karas D, Ho A, Bassett AS. Functional outcomes of adults with 22q11.2 deletion syndrome. Genet Med 2012;14:836843.

17. Ferrell RB, Wolinsky EJ, Kauffman Cl, Flashman LA, McAllister TW. Neuropsychiatric syndromes in adults with intellectual disability: issues in assessment and treatment. Curr Psychiatry Rep 2004;6:380-390.

18. Tang $S X, Y i J J$, Calkins ME, et al. Psychiatric disorders in 22q11.2 deletion syndrome are prevalent but undertreated. Psychol Med 2014;44:1267-1277.

19. Bassett AS, Chow EW. Schizophrenia and 22q11.2 deletion syndrome. Curr Psychiatry Rep 2008;10:148-157.

20. Bassett AS, Scherer SW, Brzustowicz LM. Copy number variations in schizophrenia: critical review and new perspectives on concepts of genetics and disease. Am J Psychiatry 2010;167:899-914.

21. van Amelsvoort $T$, Henry J, Morris $R$, et al. Cognitive deficits associated with schizophrenia in velo-cardio-facial syndrome. Schizophr Res 2004;70:223-232.

22. Bassett AS, Chow EW, AbdelMalik P, Gheorghiu M, Husted J, Weksberg R. The schizophrenia phenotype in 22q11 deletion syndrome. Am J Psychiatry 2003:160:1580-1586.

23. Chow EW, Watson M, Young DA, Bassett AS. Neurocognitive profile in $22 \mathrm{q} 11$ deletion syndrome and schizophrenia. Schizophr Res 2006;87:270-278.

24. Stoddard J, Niendam T, Hendren R, Carter C, Simon TJ. Attenuated positive symptoms of psychosis in adolescents with chromosome 22q11.2 deletion syndrome. Schizophr Res 2010;118:118-121.
25. Yuen T, Chow EW, Silversides CK, Bassett AS. Premorbid adjustment and schizophrenia in individuals with 22q11.2 deletion syndrome. Schizophr Res 2013;151:221-225.

26. Butcher N, Fung WLA, Fitzpatrick $L$, et al. Response to clozapine in a clinically identifiable subtype of schizophrenia: 22q11.2 deletions mediate side effect risk and dosage. Br J Psychiatry, in press.

27. Bassett AS, Chow EWC, Hodgkinson KA. Genetics of schizophrenia and psychotic disorders. In: Smoller JW, Sheidley BR, Tsuang MT (eds). Psychiatric Genetics: Applications in Clinical Practice. American Psychiatric Publishing: Washington, DC, 2008:99-130.

28. Antshel KM, Shprintzen R, Fremont W, Higgins AM, Faraone SV, Kates WR. Cognitive and psychiatric predictors to psychosis in velocardiofacial syndrome: a 3-year follow-up study. J Am Acad Child Adolesc Psychiatry 2010:49:333-344.

29. Gothelf D, Schaer M, Eliez S. Genes, brain development and psychiatric phenotypes in velo-cardio-facial syndrome. Dev Disabil Res Rev 2008;14:59-68.

30. Vorstman JA, Breetvelt E, Duijff SN, et al. A decline in verbal intelligence precedes the onset of psychosis in patients with the $22 q 11.2$ deletion syndrome. JAMA Psychiatry, in press.

31. Martin N, Mikhaelian M, Cytrynbaum C, et al. 22q11.2 deletion syndrome: attitudes towards disclosing the risk of psychiatric illness. J Genet Couns 2012:21:825-834.

32. Bassett AS, Marshall CR, Lionel AC, Chow EW, Scherer SW. Copy number variations and risk for schizophrenia in 22q11.2 deletion syndrome. Hum Mol Genet 2008;17:4045-4053.

33. Vorstman JA, Breetvelt EJ, Thode KI, Chow EW, Bassett AS. Expression of autism spectrum and schizophrenia in patients with a 22q11.2 deletion. Schizophr Res 2013:143:55-59.

34. Swillen A, Vandeputte L, Cracco J, et al. Neuropsychological, learning and psychosocial profile of primary school aged children with the velo-cardio-facial syndrome (22q11 deletion): evidence for a nonverbal learning disability? Child Neuropsychol 1999:5:230-241.

35. Swillen A, Vogels A, Devriendt K, Fryns JP. Chromosome $22 q 11$ deletion syndrome: update and review of the clinical features, cognitive-behavioral spectrum, and psychiatric complications. Am J Med Genet 2000;97:128-135.

36. De Smedt B, Swillen A, Verschaffel L, Ghesquière P. Mathematical learning disabilities in children with 22q11.2 deletion syndrome: a review. Dev Disabil Res Rev 2009:15:4-10.

37. Henry JC, van Amelsvoort T, Morris RG, Owen MJ, Murphy DG, Murphy KC. An investigation of the neuropsychological profile in adults with velo-cardio-facial syndrome (VCFS). Neuropsychologia 2002;40:471-478.

38. Kao A, Mariani J, McDonald-McGinn DM, et al. Increased prevalence of unprovoked seizures in patients with a 22q11.2 deletion. Am J Med Genet $A$ 2004;129A:29-34.

39. Andrade DM, Krings T, Chow EW, Kiehl TR, Bassett AS. Hippocampal malrotation is associated with chromosome 22q11.2 microdeletion. Can J Neurol Sci 2013;40:652-656.

40. Ryan AK, Goodship JA, Wilson DI, et al. Spectrum of clinical features associated with interstitial chromosome 22 q11 deletions: a European collaborative study. J Med Genet 1997:34:798-804.

41. Cheung EN, George SR, Andrade DM, Chow EW, Silversides CK, Bassett AS. Neonatal hypocalcemia, neonatal seizures, and intellectual disability in 22q11.2 deletion syndrome. Genet Med 2014;16:40-44.

42. González W, Bautista RE. Seizures and EEG findings in an adult patient with DiGeorge syndrome: a case report and review of the literature. Seizure 2009:18:648-651.

43. Choi JH, Shin YL, Kim GH, et al. Endocrine manifestations of chromosome 22q11.2 microdeletion syndrome. Horm Res 2005;63:294-299.

44. Cuneo BF, Driscoll DA, Gidding SS, Langman CB. Evolution of latent hypoparathyroidism in familial 22q11 deletion syndrome. Am J Med Genet 1997;69:50-55.

45. Kar PS, Ogoe B, Poole R, Meeking D. Di-George syndrome presenting with hypocalcaemia in adulthood: two case reports and a review. J Clin Pathol 2005:58:655-657.

46. Cheung EN, George SR, Costain GA, et al. Prevalence of hypocalcaemia and its associated features in 22q11.2 deletion syndrome. Clin Endocrinol (Oxf) 2014;81:190-196.

47. Brauner R, Le Harivel de Gonneville A, Kindermans C, et al. Parathyroid function and growth in 22q11.2 deletion syndrome. J Pediatr 2003;142:504-508.

48. Kawame $\mathrm{H}$, Adachi $\mathrm{M}$, Tachibana $\mathrm{K}$, et al. Graves' disease in patients with 22q11.2 deletion. J Pediatr 2001;139:892-895. 
49. Guzman ML, Delgado I, Lay-Son G, Willans E, Puga A, Repetto GM. Growth in Chilean infants with chromosome $22 q 11$ microdeletion syndrome. Am J Med Genet A 2012;158A:2682-2686.

50. Gennery AR. Immunological aspects of $22 q 11.2$ deletion syndrome. Cell Mol Life Sci 2012;69:17-27.

51. Sullivan KE, Jawad AF, Randall $P$, et al. Lack of correlation between impaired $T$ cell production, immunodeficiency, and other phenotypic features in chromosome 22q11.2 deletion syndromes. Clin Immunol Immunopathol 1998;86:141-146.

52. Matsuoka R, Kimura M, Scambler PJ, et al. Molecular and clinical study of 183 patients with conotruncal anomaly face syndrome. Hum Genet 1998;103:70-80.

53. Bretelle F, Beyer L, Pellissier MC, et al. Prenatal and postnatal diagnosis of 22q11.2 deletion syndrome. Eur J Med Genet 2010;53:367-370.

54. John AS, McDonald-McGinn DM, Zackai EH, Goldmuntz E. Aortic root dilation in patients with 22q11.2 deletion syndrome. Am J Med Genet A 2009;149A:939-942.

55. Warnes CA, Williams RG, Bashore TM, et al.; American College of Cardiology; American Heart Association Task Force on Practice Guidelines (Writing Committee to Develop Guidelines on the Management of Adults With Congenital Heart Disease); American Society of Echocardiography; Heart Rhythm Society; International Society for Adult Congenital Heart Disease; Society for Cardiovascular Angiography and Interventions; Society of Thoracic Surgeons. ACC/AHA 2008 guidelines for the management of adults with congenital heart disease: a report of the American College of Cardiology/American Heart Association Task Force on Practice Guidelines (Writing Committee to Develop Guidelines on the Management of Adults With Congenital Heart Disease). Developed in Collaboration With the American Society of Echocardiography, Heart Rhythm Society, International Society for Adult Congenital Heart Disease, Society for Cardiovascular Angiography and Interventions, and Society of Thoracic Surgeons. J Am Coll Cardiol 2008;52:e143-e263.

56. Silversides CK, Kiess M, Beauchesne L, et al. Canadian Cardiovascular Society 2009 Consensus Conference on the management of adults with congenital heart disease: outflow tract obstruction, coarctation of the aorta, tetralogy of Fallot, Ebstein anomaly and Marfan's syndrome. Can J Cardio/ 2010;26:e80-e97.

57. Gatzoulis MA, Walters J, McLaughlin PR, Merchant N, Webb GD, Liu P. Late arrhythmia in adults with the mustard procedure for transposition of great arteries: a surrogate marker for right ventricular dysfunction? Heart 2000;84:409-415.

58. Ghai A, Silversides C, Harris L, Webb GD, Siu SC, Therrien J. Left ventricular dysfunction is a risk factor for sudden cardiac death in adults late after repair of tetralogy of Fallot. J Am Coll Cardiol 2002;40:1675-1680.

59. Hickey EJ, Veldtman G, Bradley TJ, et al. Late risk of outcomes for adults with repaired tetralogy of Fallot from an inception cohort spanning four decades. Eur J Cardiothorac Surg 2009;35:156-164; discussion 164.
60. Costain G, Chow EW, Silversides CK, Bassett AS. Sex differences in reproductive fitness contribute to preferential maternal transmission of $22 q 11.2$ deletions. J Med Genet 2011;48:819-824.

61. Tarquinio DC, Jones MC, Jones KL, Bird LM. Growth charts for $22 q 11$ deletion syndrome. Am J Med Genet A 2012;158A:2672-2681.

62. Besseau-Ayasse J, Violle-Poirsier C, Bazin A, et al. A French collaborative survey of 272 fetuses with 22q11.2 deletion: ultrasound findings, fetal autopsies and pregnancy outcomes. Prenat Diagn 2014;34:424-430.

63. Hatton R, Colman JM, Sermer M, Grewal J, Silversides CK. Cardiac risks and management of complications in pregnant women with congenital heart disease. Future Cardiol 2012;8:315-327.

64. Costain G, Chow EW, Ray PN, Bassett AS. Caregiver and adult patient perspectives on the importance of a diagnosis of $22 q 11.2$ deletion syndrome. $J$ Intellect Disabil Res 2012;56:641-651.

65. Krahn LE, Maraganore DM, Michels VV. Childhood-onset schizophrenia associated with parkinsonism in a patient with a microdeletion of chromosome 22. Mayo Clin Proc 1998;73:956-959.

66. Booij J, van Amelsvoort T, Boot E. Co-occurrence of early-onset Parkinson disease and 22q11.2 deletion syndrome: potential role for dopamine transporter imaging. Am J Med Genet A 2010;152A:2937-2938.

67. Butcher N, Kiehl T, Hazrati L, et al. Individuals with 22q11.2 deletion syndrome are at increased risk of early-onset Parkinson disease: identification of a novel genetic form of Parkinson disease and its clinical implications. JAMA Neurol 2013:1359-1366.

68. Bassett AS, Chow EW, Husted J, et al. Premature death in adults with 22q11.2 deletion syndrome. J Med Genet 2009;46:324-330.

69. Gerdes M, Solot C, Wang PP, McDonald-McGinn DM, Zackai EH. Taking advantage of early diagnosis: preschool children with the 22 q11.2 deletion. Genet Med 2001;3:40-44.

70. McDonald-McGinn DM, Zackai EH. Genetic counseling for the $22 q 11.2$ deletion. Dev Disabil Res Rev 2008;14:69-74.

71. Vantrappen G, Devriendt K, Swillen A, et al. Presenting symptoms and clinical features in 130 patients with the velo-cardio-facial syndrome. The Leuven experience. Genet Couns 1999;10:3-9.

72. Digilio MC, Angioni A, De Santis M, et al. Spectrum of clinical variability in familial deletion 22q11.2: from full manifestation to extremely mild clinical anomalies. Clin Genet 2003;63:308-313.

73. Devriendt K, Swillen A, Fryns JP, Proesmans W, Gewillig M. Renal and urological tract malformations caused by a $22 q 11$ deletion. J Med Genet 1996;33:349.

74. Finucane B. Genetic counseling for women with intellectual disabilities. In: LeRoy BS, Veach PM, Bartels DM (eds). Genetic Counseling Practice: Advanced Concepts and Skills. Wiley-Blackwell: Hoboken, NJ, 2010: 281-303. 